\title{
Abiotic Elicitors Involved in Plant Phe nolics Synthesis
}

\author{
Raluca Alexandra* \\ Universidad de Las Américas, USA
}

Submission: April 18, 2018; Published: May 04, 2018

"Corresponding author: Raluca Alexandra, Universidad de Las Américas, USA, Email: rmihai@espe.edu.ec

\section{Introduction}

The plant kingdom represents an enormous reservoir of chemical compounds with beneficial effects for the plant systems and for the human health. One of these representative compounds are the phenolics which play important roles in plant development, provide structural integrity, scaffolding support to plants and protection against stress. These compounds receive an increasing attentions due to their bioactivity related to the antioxidant behaviour attributed to the ability to chelate metals, plant secondary metabolites represent important constituents of the human diet which have been associated with the inhibition of atherosclerosis and cancer and also with the prevention of certain skin disorders. For these reason and due to their natural origin and low toxicity, phenolic compounds are a promising tool in eliminating the causes and effects of potentially lifethreatening diseases.

The evolving commercial importance of phenolics secondary metabolites has in recent years resulted in a great interest for the possibility of production of these bioactive plant metabolites by means of tissue culture technology. Plant cell and tissue culture technologies can be established routinely under sterile conditions from explants, such as plant leaves, stems, roots, and meristems for both the ways for multiplication and extraction of secondary metabolites. In vitro production of phenolics secondary metabolite in plant cell cultures has been reported from various medicinal plants, in order to their commercial production. The utilization of plant cells for the production of natural or recombinant compounds of commercial interest

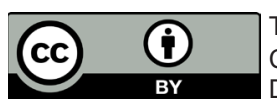

This work is licensed under Creative

Commons Attribution 4.0 License

DOI: 10.19080/ARTOAJ.2018.15.555969 inhibition of lipoxygenase and scavenge of free radicals. These

has gained increasing attention over past decades The current yield and productivity cannot fulfil the commercial goal of plant cell-based bioprocess for the production of most secondary metabolites. In order to stretch the boundary, new strategies to improve the production of secondary metabolites must be considered. One of these is represented by the use of biotic and abiotic elicitors, compounds triggering the formation of secondary metabolites. The present study aimed to investigate the use of abiotic elicitors mannitol, thidiazuron and abscisic acid as a posible reliable tool for the production of phenolic compounds in babaco (Vasconcellea $\times$ Heilbornii cv.), a plant with latin american origin. Based on this lime light, the present review is aimed to elicitors include there is an increased interest in these compounds because they have been associated with the inhibition of atherosclerosis and cancer. The bioactivity of phenolics may be Plant phenolic compounds such as flavonoids and lignin precursors are. These dietary phytophenolics have been recognized largely as beneficial antioxidants that can scavenge harmful active oxygen species including $\mathrm{O}_{2}^{-}, \mathrm{H}_{2} \mathrm{O}_{2}$, $\mathrm{OH}$, and $(1)_{2}$. In plant systems, phytophenolics can act as antioxidants by donating electrons to guaiacol-type peroxidases (GuPXs) for the detoxification of $\mathrm{H}_{2} \mathrm{O}_{2}$ produced under stress conditions has received due to their diverse phytometabolic cont. Due to their natural origin and low toxicity, phenol 0063 compounds are a promising tool in eliminating the causes and effects of skin aging, skin diseases, and skin damage, including wounds and burns. Polyphenols, both embarrassing minor problems (e.g., wrinkles, acne) or serious, such as cancer.

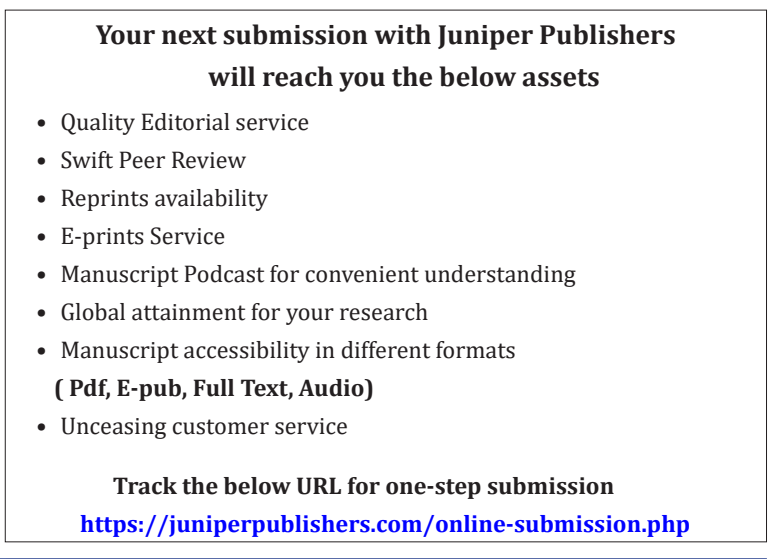

